\title{
An unusual abnormality of human spermatozoa
}

\author{
Elizabeth Aughey and P. S. Orr* \\ Department of Veterinary Anatomy, University of Glasgow, and \\ * Department of Urology, Glasgow Royal Infirmary, Glasgow, U.K.
}

\begin{abstract}
Summary. A defect affecting $90 \%$ of the spermatozoa of an infertile man consisted of round heads and a loss of tails, probably caused by a developmental abnormality at the spermatid stage.
\end{abstract}

\section{Introduction}

Pleomorphism is a characteristic of spermatozoa from fertile men (McLeod \& Gold, 1951), and it is therefore difficult to categorize specific abnormal spermatozoa in man unless they are present as the predominant type in one individual (Ross, Christie \& Kerr, 1971). Sperm samples with a high proportion of a single abnormality are extremely uncommon. The case reported in the present paper showed a high proportion of abnormal spermatozoa of one type.

\section{Material and Methods}

A 33-year-old male presented at the Male Subfertility Clinic after 6 years of involuntary infertility. He suffered from ulcerative colitis and had been treated with sulphasalazine (Salazopyrin: Pharmacia) for 5 years. His haemoglobin, serum bilirubin and red cell folate levels were within normal limits. The patient's wife had been investigated for infertility and found to be normal. Seminal analysis on several occasions showed a sperm density ranging from $25-78 \times 10^{6}$ spermatozoa $/ \mathrm{ml}$ and a sperm motility of $5-20 \%$. The patient had a normal male phenotype. Mitotic chromosome analysis showed a normal male karyotype of 46 chromosomes. Meiotic analysis revealed a normal pattern of 23 bivalents. Plasma levels of testosterone and $\mathrm{LH}$ were normal. The semen was examined with the light microscope and $90 \%$ of the spermatozoa were abnormal with round heads. Most of these spermatozoa had a detached tail which was, however, still motile.

Concentrated pellets of spermatozoa were prepared for electron microscopy after centrifugation of the semen sample. The pellets were fixed in buffered paraformaldehyde at pH $7 \cdot 4$ (Peters \& Palay, 1966), dehydrated in ascending grades of acetone and embedded in Spurr's resin (Luft, 1961). Thick sections were cut at $1 \mu \mathrm{m}$ and stained with Toluidine blue, and selected areas were then cut at 60-70 $\mathrm{nm}$, stained routinely with uranyl acetate/lead citrate and examined with the Hitachi HS8 electron microscope.

\section{Results and Discussion}

Examination of the thick sections showed areas of round headed spermatozoa, separate tails and cellular debris (Pl. 1, Fig. 1).

With the electron microscope, the abnormal round-headed spermatozoa (Pl. 1, Fig. 2) often showed eccentric implantation plates, double tails and thickened mid-pieces. The mid-piece mitochondrial helix did not have a regular pattern and excessive amounts of cytoplasm containing membrane whorls were observed. The decapitated sperm defect with separation of the spermatozoa into loose heads and tails has been described in Guernsey bulls in which it causes a congenital sterility 
(Hancock \& Rollinson, 1949). The ultrastructural features of the mid-piece and tail of ejaculated spermatozoa from such sterile Guernsey bulls (Blom \& Birch-Anderson, 1970) are similar to those described here for a man. However, head defects, in the form of uncondensed chromatin, and poorly formed acrosomes were also evident in this patient in the majority of spermatozoa in each sample.

Examination of a testicular biopsy showed disorganization of the normal clonal pattern of development in many of the seminiferous tubules (PI. 1, Figs 3 and 4). The few secondary spermatocytes and late stage spermatids present were dispersed randomly throughout the tubular epithelium. Electron microscope examination of the testicular biopsy confirmed the lack of normal spermatozoa. Most of the developing spermatids had eccentric implantation plates, often without an accompanying centriole and there was no regular mitochondrial arrangement. In the large number of sections examined, many spermatids did not have any tail development at all. It is possible that this was due to the relatively small area of the seminiferous epithelium examined, but the regular appearance of these tail-less or eccentric implantation sites suggests that the primary defect is of tail formation in the spermatid. The aetiology of the abnormality is unknown.

Subfertility in the human male is relatively common and accounts for about $20-40 \%$ of infertile marriages (Hendry, 1975). Apart from primary gonadal failure, hormonal causes are seldom found to account for the infertility. Chromosomal defects as a cause of reduced fertility, such as Klinefelter's Syndrome with an XXY karyotype, is well recognized but infrequently seen. The majority of patients with subfertility and a low seminal count show arrest of spermatogenesis or 'sloughing' of the seminiferous tubules (Bobrow, 1973). The causes of these defects are not understood.

Sperm abnormalities detectable at ultrastructural level are probably a more common cause of infertility than has been previously suggested and routine use of electron microscope examination would therefore seem indicated.

\section{References}

Boвrow, L.G. (1973) Testicular biopsy. Recent $A d v$. Clin. Path. 6, 123-131.

Blom, E. \& Birch ANderson, A. (1970) Ultrastructure of the 'decapitated sperm defect' in Guernsey bulls. J. Reprod. Fert. 23, 67-72.

Hancock, J.L. \& Rollinson, D.H.L. (1949) A seminal defect associated with the sterility of Guernsey bulls. Vet. Rec. 61, 742-743.

Hendry, W.F. (1975) Male infertility. Practitioner 214, 60-69.

LUFT, J.H. (1961) Improvements in epoxy resin embedding methods. J. Biophys. Biochem. Cytol.9, 409-414.
McLeod, J. \& GoLd, R.A. (1951) An analysis of the motile activity in the spermatozoa of 1,000 fertile men and 1,000 men in infertile marriages. Fert. Steril. 2, 187-204.

Peters, A. \& Palay, S.L. (1966) The morphology of the lamina $A$ and $A \mid$ of the dorsal nucleus of the lateral geniculate body of the cat. J. Anat. 100, 451-468.

Ross, A., Christie, S. \& Kerr, M.G. (1971) An electron microscope study of a tail abnormality in spermatozoa from a subfertile man. J. Reprod. Fert. 24, 99-103.

Received 23 November 1977

\section{EXPLANATION OF PLATE 1}

Fig. 1. The predominance of round-headed spermatozoa (arrowed) with separation of the tails (T) is seen in this $1 \mu \mathrm{m}$ toluidine blue section of a semen sample pellet.

Fig. 2. Electron micrograph of an abnormal human spermatozoon showing the round immature head (H), the acrosome (A) and malformed tailpiece with vesicles (V) and mitochondrial membranes (M).

Fig. 3. $1 \mu \mathrm{m}$ Toluidine blue-stained Epon section of the testicular biopsy showing the disorganized seminiferous epithelium, fluid-filled spaces (F) and randomly scattered primary spermatocytes (arrowed). Some peritubular fibrosis is evident (BM).

Fig. 4. $1 \mu \mathrm{m}$ Toluidine blue-stained Epon section from a 'normal' area of seminiferous epithelium in another patient showing the closely organized cells and developing spermatozoa (arrowed). Some peritubular fibrosis is evident (BM). 

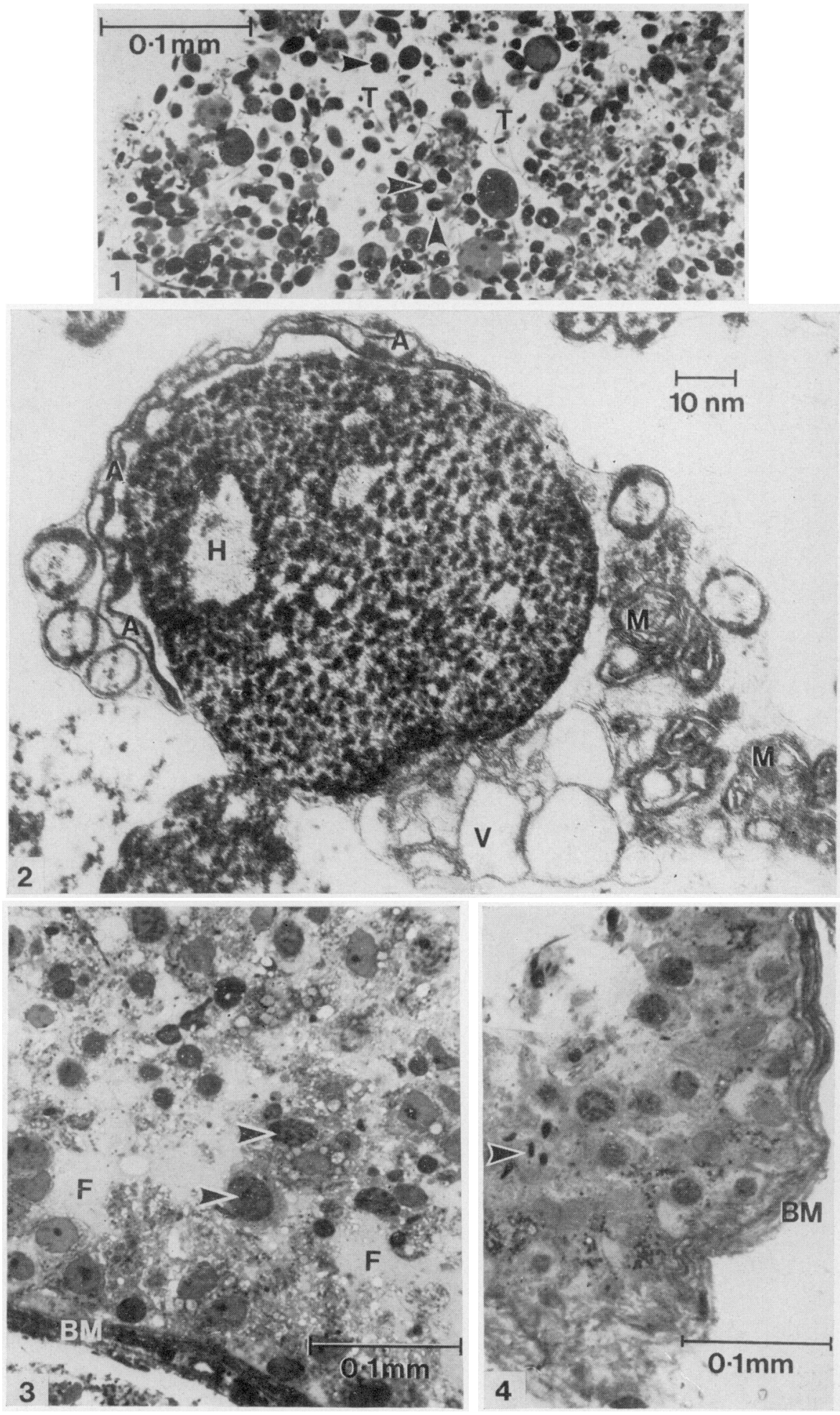

(Focing f. 342) 